\title{
Proteus and coliform meningoencephalitis in neonates
}

\author{
W. R. SHORTLAND-WEBB
}

From the Children's Hospital, Birmingham

SYNOPSIS The characteristic necropsy and histological appearances are described of nine cases of Proteus meningoencephalitis in neonates. One case which was not due to Proteus has been included because of the close similarity of the gross appearances of the brain. Umbilical sepsis in half the cases indicated that this is a common portal of entry of these organisms. That epidemiological factors may be of importance in the aetiology was suggested by the distribution of cases.

Proteus organisms are widely distributed in nature. They play an important role in the decay of organic matter of animal origin, including sewage, and exist as saprophytes in the alimentary tract of man. Under certain circumstances they may become pathogenic and in particular they may infect the urinary tract, the ear, and burns and scalds. Meningitis due to Proteus is extremely uncommon, and in adults and older children it appears to be limited to a few cases following otitis media and sinus thrombosis (Kaplan and Poweleit, 1949); otherwise it occurs almost exclusively in neonates though it is still a rarity even at this relatively susceptible age. The few reported cases indicate that neonatal meningitis due to Proteus is an extremely lethal condition and this is also the experience in Birmingham. The purpose of this paper is to describe the characteristic findings at necropsy and on histological examination and to comment on the aetiology.

\section{CASE REPORTS}

CASE 1 A male infant was born at 33 weeks to a para 2 mother aged 23 years. There was hyperemesis but pregnancy was otherwise unremarkable. Spontaneous vertex delivery followed an uncomplicated labour lasting 12 hours. At birth the infant weighed 1,814 g and was in fair general condition. The onset of respiratory distress a few hours later necessitated transfer to a premature baby unit where the infant was noticed to have a feeble cry and a rash on his chest. Gradual improvement followed treatment in humidified oxygen, the removal of $12 \mathrm{ml}$ of blood by umbilical catheter, digitalization, achromycin, and chloramphenicol. On the second day the blood sugar level was $70 \mathrm{mg} \%$ and the serum bilirubin $8.6 \mathrm{mg} \%$; blood culture grew Proteus

Received for publication 25 October 1967. mirabilis which were sensitive to streptomycin, chloro- $\complement$ mycetin, neomycin, albamycin, and partially sensitive to novobiocin. On the third day he became pale, cyanosed, $\vec{\varphi}$ jaundiced, tense and rigid, and had a squeaky cry; he $\infty$ died a few hours later aged 65 hours.

Necropsy was performed 91 hours after death. The body measurements corresponded to 33 to 34 weeks' gestation; weight $1,590 \mathrm{~g}$, length $43 \mathrm{~cm}$, head circumference $29 \mathrm{~cm}$.

The cranial cavity had a foul smell and both cerebral hemispheres were greyish in colour and grossly softened, much of the cerebral substance having the consistency of thick sauce. There was extensive, bilateral, subdural haemorrhage with no tentorial tears, and both occipital poles showed localized subarachnoid haemorrhages. The hindbrain appeared normal.

There was widespread congestion and collapse of both lungs with subpleural haemorrhages and bloodstained froth in the bronchi.

Bacteriology Swabs taken from the left and right cerebral hemispheres grew Proteus mirabilis. Proteus was present in small numbers in a portion of the right $ᄋ$ lower lobe of lung; cultures on solid media failed to grow this organism but subcultures of a glucose broth culture gave a growth of Proteus mirabilis.

Histology The periventricular cerebral tissue showed $N$ extensive necrosis with haemorrhage and intravascular $\mathrm{N}$ haemolysis but no inflammatory reaction. The lungs showed a little granular eosinophilic debris and mono- $\omega$ nuclear cells within the alveolar ducts.

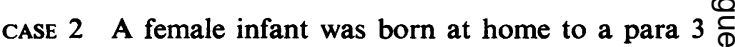
mother. Pregnancy was uneventful but delivery was precipitate. The birth weight was $2,722 \mathrm{~g}$. Towards the end of the first month of life the infant began to refuse her feeds. On admission to the Birmingham Children's $\underset{D}{\mathbb{D}}$ Hospital she was an ill, wasted, cold infant with a rectal temperature of $25^{\circ} \mathrm{C}\left(77.0^{\circ} \mathrm{F}\right)$. She showed peripheral $\stackrel{\mathbb{Q}}{2}$ cyanosis, had a feeble cry, swollen eyelids, candidal $\sigma$ infection of the mouth, and a napkin rash. The pulse 
rate was $40 /$ minute and the heart rate was $88 /$ minute and irregular. Respirations were shallow, the respiratory rate was $32 /$ minute, and there was dullness and diminished air entry at the right base. Treatment included penicillin, streptomycin, nystatin, and hydrocortisone. After eight hours' slow warming the rectal temperature was $31.7^{\circ} \mathrm{C}\left(89 \cdot 0^{\circ} \mathrm{F}\right)$. Attacks of apnoea and cyanosis occurred approximately every three hours and she died 13 hours following admission.

Necropsy was performed $11 \frac{1}{2}$ hours after death. The body weighed $2,394 \mathrm{~g}$, measured $47.5 \mathrm{~cm}$ in length, and had a head circumference of $33 \mathrm{~cm}$. There was moderate wasting, superficial ulceration of the lower lip, and a slight napkin rash. On opening the cranial cavity there was a foul smell but there was no gross evidence of meningitis or haemorrhage. The brain was soft and swollen with a dull, purple-black discoloration over the left temporo-parietal region, and the cerebral white matter was greyish-pink in appearance. The lungs showed generalized collapse and congestion. There was thoracic scoliosis due to hemivertebrae.

Bacteriology A swab of the left cerebral hemisphere showed a heavy growth of proteus and a few Klebsiella; the lung showed a heavy growth of Klebsiella together with a few Proteus and viridans streptococci; the surface of the pour plate of heart blood was covered with Proteus; subculture of the liquid glucose broth fluid culture grew Klebsiella with a few Proteus vulgaris and viridans streptococci. Isolation of these organisms suggested that this postmortem blood culture might have been contaminated.

Histology The brain and spinal cord showed almost generalized infiltration with filamentous, Gram-negative bacilli (proteus); this was most marked in the areas noted to be softened at necropsy and was least in the lower spinal cord. There was no accompanying inflammatory cell reaction even in the meninges, nor was there any vascular lesion in spite of the fact that the bacteria were often seen to colonize the small blood vessels. There were, however, many swollen microglia which on frozen sections were shown to contain sudanophilic material. Neurones in the cortex of the softened areas showed shrinkage of cytoplasm and homogeneous, deeply basophilic nuclei. The lungs showed acute bronchopneumonia in two lobes.

CASE 3 A male infant was born at 38 weeks to a para 3, Rhesus-negative mother. Delivery was complicated by placenta praevia and antepartum haemorrhage. Birth weight was 2,381 $\mathrm{g}$. The cord blood was group $\mathrm{A}$, Rhesus-positive, Coombs test positive. Haemoglobin was $98 \%$ and the bilirubin $3.1 \mathrm{mg} \%$. The general condition of the infant was poor and he developed jaundice with a maximum bilirubin level of $20.0 \mathrm{mg} \%$ on the fifth day. During the 24 hours preceding death he refused feeds and developed pyrexia, restlessness, grunting, and neck stiffness. He died on the seventh day. Lumbar puncture several hours before he died produced turbid yellow fluid which had a protein content of $325 \mathrm{mg} \%$ and contained 2,920 polymorphs, 500 lymphocytes, and 2,620 erythrocytes per $\mathrm{cmm}$. No organisms were seen in a Grarn-stained film of the centrifuged cerebrospinal fluid

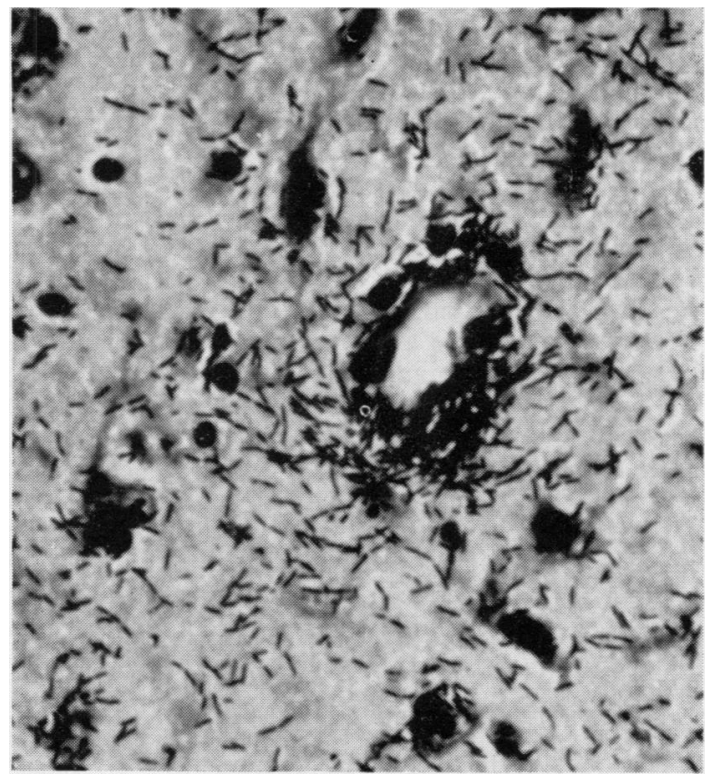

FIG. 1. Case 2. Cerebral white matter showing masses of bacilli. Giemsa $\times 1,930$.

but cultures grew Proteus sensitive to penicillin, streptomycin, chloramphenicol, novobiocin, and ampicillin, and also coagulase-negative staphylococci. It was suggested that these latter organisms might have been contaminants.

Necropsy was performed four days after death. The body was moderately jaundiced, weighed $2,040 \mathrm{~g}$, measured $47 \mathrm{~cm}$, and had a head circumference of 30.5 $\mathrm{cm}$. The umbilicus was clean but there was an erythematous, slightly haemorrhagic rash on the trunk. The cerebral hemispheres were brownish, soft, swollen, and foul smelling. A thick layer of exudate covered the posterior aspect of the cerebellum but there was none surrounding the spinal cord. The brain showed extensive softening and reddening on slicing; there was no kernicterus of the brain stem or cerebellar nuclei but there was slight yellow staining of the basal nuclei. The lungs showed collapse at the bases. The liver was slightly enlarged and congested. The spleen was congested but not enlarged and gave a positive Prussian Blue reaction.

Bacteriology Swabs from the brain showed a moderate growth of Proteus. Cultures of the lung were sterile.

Histology There was marked acute inflammation of the meninges and many vessels within the brain showed perivascular cellular exudate and haemorrhage or were necrotic and filled with fibrin thrombus. Inflammatory cells, mainly mononuclear, extended into surrounding brain tissue in which much of the cerebral white matter was necrotic. There was much proliferation and swelling of glial cells. There were abundant Gram-negative rods, especially in the perivascular infiltrates. The lungs showed alveolar oedema but no pneumonia. There was haemosiderosis of the liver and spleen with only a small amount of erythropoiesis. 
CASE 4 A male infant was born at 30 weeks to a primiparous mother aged 26 years. Pregnancy and labour were normal. He weighed 1,134 $\mathrm{g}$ at birth, and because of grunting respirations was admitted to a premature baby unit. He was nursed in an incubator and fed on expressed mother's milk. On the third day he became jaundiced and on the following day there was an offensive umbilical discharge. He died at 92 hours following frequent apnoeic attacks. Blood culture showed a mixture of $E$. coli and Proteus; both organisms were insensitive to penicillin and tetracyclines but sensitive to streptomycin and chloramphenicol.

Necropsy was performed six and a half hours after death. The body was that of a moderately jaundiced, premature infant of estimated maturity of 29 weeks. Weight was $975 \mathrm{~g}$, length $37 \mathrm{~cm}$, head circumference $27 \mathrm{~cm}$. Both cerebral hemispheres were represented by extremely soft, chocolate-coloured tissue with a foul smell but meningeal exudate was not seen. The lungs showed diffuse congestion and collapse. The umbilical vein appeared normal but both umbilical arteries were filled with pus.

Bacteriology Proteus of the same serological type isolated during life was found on culture of brain, lung, and umbilical arteries.

Histology There was extensive necrosis of the brain with intravascular haemolysis but no inflammatory reaction. All areas, including apparently normal brain and meninges, showed abundant Gram-negative rods. The lungs showed congestion and intraalveolar haemorrhage with no inflammatory change. The intraabdominal segments of the umbilical arteries contained septic thrombus; one showed necrosis of the intima with many Gram-negative rods but no cellular reaction.

CASE 5 A female infant was delivered spontaneously by the vertex at 32 weeks' gestation to a 32-year-old para 2 mother. There was antepartum haemorrhage. The infant weighed $1,474 \mathrm{~g}$ and required oxygen by mask. On transfer to a premature baby unit when $3 \frac{1}{2}$ hours old she had grunting respirations and cyanosis. Jaundice was noticed on the third day when the plasma bilirubin level was $10.3 \mathrm{mg} \%$. On the fifth day she developed pallor and apnoeic attacks and she died the following day. Treatment included humidified oxygen by mask during cyanotic episodes, terramycin, kanamycin, and caffeine sodium benzoate.

Necropsy was performed five days after death. The body was that of a premature infant with an estimated maturity of 31 weeks. Weight was $1,237 \mathrm{~g}$, length 39.5 $\mathrm{cm}$, head circumference $28 \mathrm{~cm}$. On opening the cranial cavity there was a foul smell. The brain was purpleblack, discoloured, and extensively softened but there was no gross meningeal exudate. Both umbilical arteries contained pus, the right more than the left.

Bacteriology Proteus vulgaris was cultured from brain, umbilicus, lung, and heart blood.

Histology The cerebral white matter particularly showed gross haemorrhagic disorganization with haemolysis of red cells. The architecture of the hindbrain and cerebral cortex was fairly well preserved but the nerve cells generally were shrunken with intensely basophilic

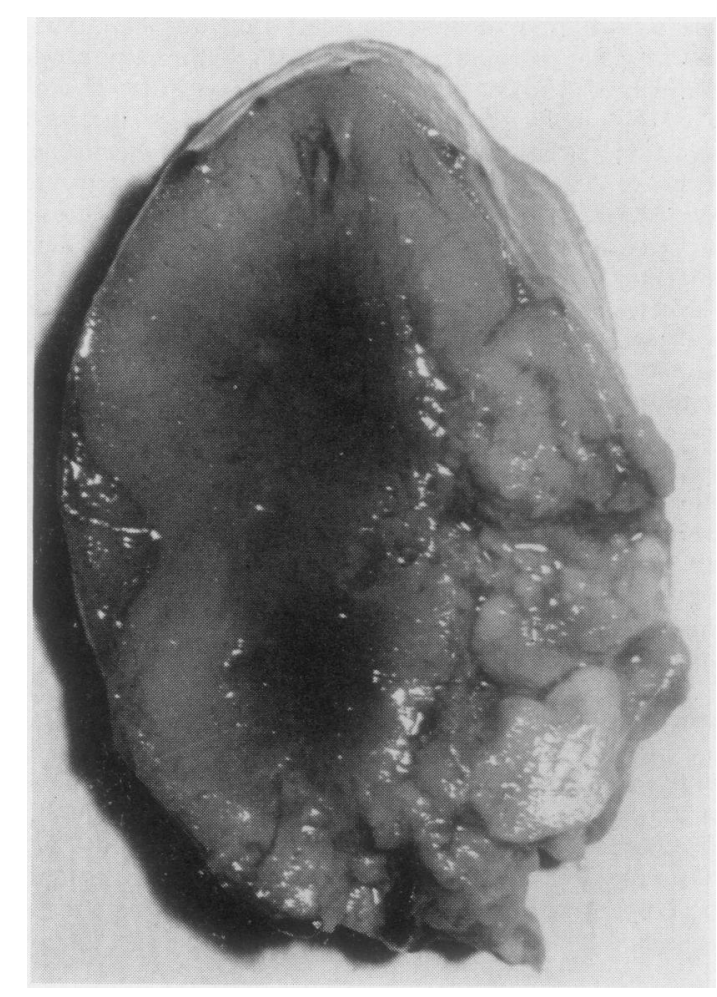

FIG. 2. Case 5. Coronal slice of left cerebral hemisphere showing complete blackening and necrosis.

nuclei. The meninges, particularly over the ventral surface of the hindbrain, showed an exudate of fibrin, polymorphs, and mononuclear cells. All sections showed abundant Gram-negative bacilli; in the brain stem these were particularly numerous in the descending corticospinal tracts and intra-vascular haemolysis was also particularly marked in these areas, suggesting that the infection had tracked down from the cerebral hemispheres along this route. Lung sections showed haemolysis of red cells with Gram-negative rods in the walls of many blood vessels. There were focal haemorrhages but $\frac{D}{O}$ no evidence of pneumonia. Both intraabdominal umbilical arteries were filled with acute inflammatory debris $N$ and there was much oedema and infiltration with polymorphs and mononuclear cells in and around their walls. Smears from the umbilical arteries showed abun- $\mathfrak{\omega}$ dant pus cells and many Gram-negative bacilli.

CASE 6 A male infant was born to a para 1 mother. Both medical and surgical induction were employed to $\mathbb{E}$ terminate this pregnancy at 36 weeks by dates because of maternal preeclampsia and delivery was assisted by forceps. Labour lasted three and a quarter hours, the infant weighed $1,474 \mathrm{~g}$, and his general condition was fair. Two days later he was transferred from a premature baby unit to the Birmingham Children's Hospital because he failed to pass meconium and had developed signs of intestinal obstruction with a mass in the right 
loin. The blood sugar was $17 \mathrm{mg} \%$; radiological examination showed gas in the rectum and an umbilical swab showed a heavy growth of Proteus sensitive to streptomycin, neomycin, and polynoxylin. The infant was treated conservatively with intravenous fluids, penicillin, streptomycin, tetracyclines, and chloramphenicol but he died on the fourth day.

Necropsy was performed 24 hours after death. The body weighed $1,394 \mathrm{~g}$, measured $42 \mathrm{~cm}$ in length, and the head circumference was $28.5 \mathrm{~cm}$. There was much wasting and the umbilicus was reddened. On opening the cranial cavity foul-smelling gas escaped and the brain was almost completely liquefied. The meninges, dural venous sinuses, and middle ears were normal. The lungs were deep purple and partially collapsed. On opening the abdominal cavity foul-smelling gas escaped and there was fibrino-purulent peritonitis. The mesentery of the ileocaecal region was unfixed and there was a volvulus with strangulation but no perforation. The right kidney was greatly enlarged and cystic.

Bacteriology Proteus was grown in large numbers from swabs from the brain and peritoneal cavity and in small numbers from a portion of the lung.

Histology The lungs showed marked congestion, a macrophage intraalveolar exudate, and a few pus cells in the large bronchi. As the brain was completely necrotic and liquefied none was taken for histology.

CASE 7 The first of twins was born at 34 weeks to a 25-year-old gravida 2 mother. Labour lasted four hours and there was spontaneous vertex delivery of a male infant. Birth weight was $1,588 \mathrm{~g}$. He was transferred to a premature baby unit where he remained well for the first three days. He then developed a rash on the skin of the abdomen, repeated apnoeic attacks, and slight jaundice. The serum bilirubin level was $11 \mathrm{mg} \%$. At this stage Proteus vulgaris was cultured from the blood, an umbilical swab, and cerebrospinal fluid. He was treated with colomycin and tetracyclines but died aged $4 \frac{1}{2}$ days following an attack of vomiting and convulsions. Periumbilical erythema was noticed a few hours before death.

Necropsy was performed six days after death. The body weighed $1,385 \mathrm{~g}$, measured $41 \mathrm{~cm}$ in length, and had a head circumference of $28.5 \mathrm{~cm}$. The stump of the umbilical cord appeared to be clean. There was a dirty-green discoloration of the scalp and calvarium and a foul odour escaped on opening the cranial cavity. The left cerebral hemisphere was firm and blackened throughout, but the rest of the brain was soft and necrotic and altered blood covered the surface of the right cerebral hemisphere. The lungs had a slightly mottled appearance macroscopically. The peritoneal cavity contained a few millilitres of foul-smelling, turbid fluid but was otherwise unremarkable. The intima of the great vessels was stained greyish-red indicating haemolysis.

Bacteriology Meninges and lung showed a heavy growth of Proteus with very few Ps. pyocyanea. A swab from the peritoneal cavity showed a scanty pure growth of Proteus.

Histology All sections of brain showed areas of necrosis, softening, and cellular degeneration and many neurones remained as cell ghosts. The glial cells showed small, dark, irregular, pyknotic nuclei. Sections from the most severely affected areas showed complete loss of identifiable cellular contents and boundaries, cuffing of the meningeal vessels by Gram-negative rods and oval bacilli, antemortem thrombus in the lumen of some of these vessels and extensive intracerebral haemorrhage. There was little polymorph response to the infection. The lungs were moderately congested, irregularly expanded, and showed a scanty mononuclear cell, intraalveolar exudate together with occasional amniotic squames and small clumps of bacteria. There was no acute inflammatory response though some alveoli contained oedema fluid.

The other twin was normal and progressing well when last seen at the age of 14 months. The placenta was dichorionic with fused membranes and with unequal components.

CASE 8 The first of twins was born at 36 weeks to a 33-year-old para 6 Indian mother. There was hydramnios but labour and delivery were normal. The infant weighed 1,744 g and was in fair condition. Progress was unremarkable until the seventh day when there was sudden onset of grunting, convulsions, and cyanosis. He was treated with oxygen but died an hour after the onset of symptoms.

Necropsy was performed 53 hours after death. Weight was $1,685 \mathrm{~g}$, length $43 \mathrm{~cm}$, head circumference $31 \mathrm{~cm}$. The estimated maturity was 34 weeks. There was a slight but distinct pungent aroma on opening the thoracic cavity. Both lungs were markedly congested but there was no consolidation. Upon opening the cranial cavity there was a foul, rancid smell akin to that of stale fish. The meninges appeared to be oedematous but there was no gross purulent exudate. Both cerebral hemispheres, particularly the frontal lobes, were softened and discoloured purplish-black. Examination after fixation showed that there was considerable softening of both cerebral hemispheres, the cerebral peduncles, and the midbrain, together with greyish-black discoloration of the frontal and anterior part of the parietal lobes. The cerebral peduncles and the midbrain were unavoidably disrupted during removal from the skull. The hindbrain was intact and showed no obvious meningitis.

Bacteriology There were heavy growths of Proteus mirabilis from lung and meninges.

Histology Sections from the frontal necrotic tissue showed much formalin pigment and small cystic spaces filled with haemolysed red cells. The normal cortical architecture was recognizable and there was a relatively scanty leucocytic infiltration of the meninges. The occipital lobes showed no haemorrhage or haemolysis and no inflammatory exudate. A section from the midbrain showed generalized necrosis recognizable by nuclear pyknosis and loss of structural detail of the brain tissue; most vessels in this region were abnormal, some were cuffed by degenerate inflammatory cells, and many showed fibrinous material replacing the vessel wall; many of the smaller vessels showed abundant small bacilli in and around their walls easily recognizable 


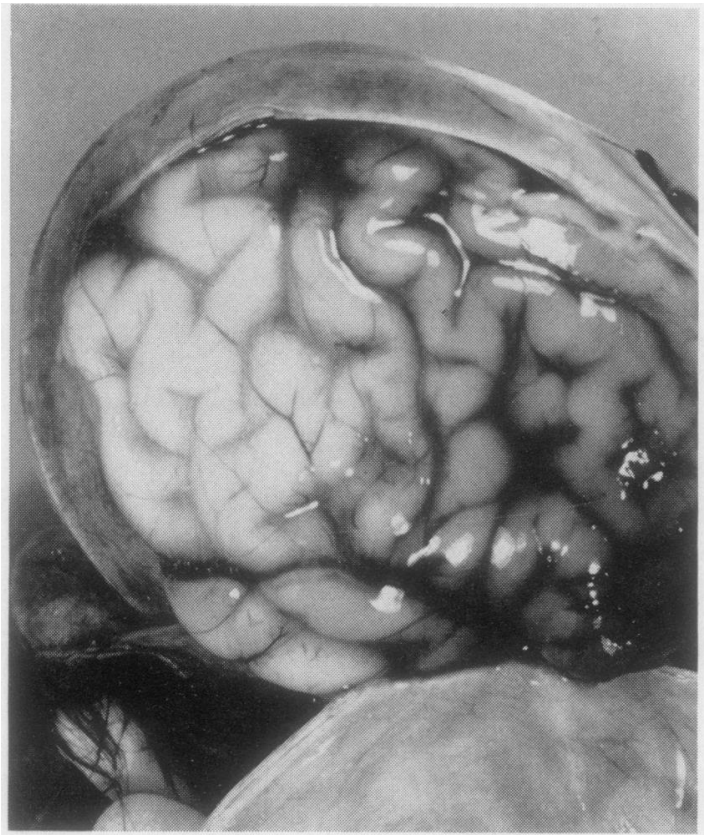

FIG. 3. Case 8. Right cerebral hemisphere in situ showing blackening of frontal and parietal lobes.

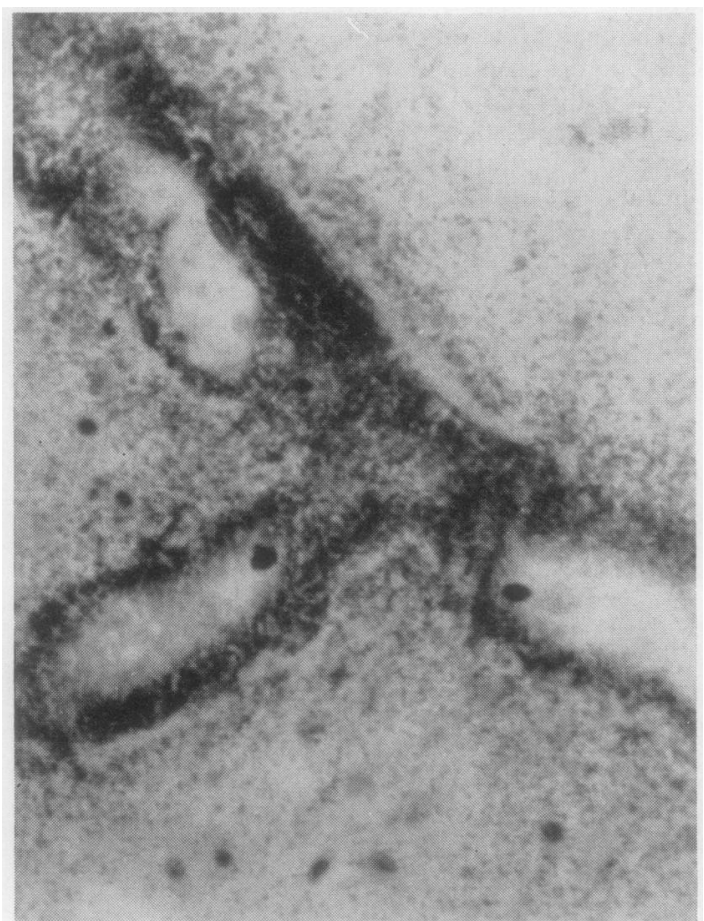

FIG. 5. Case 8. Cerebral white matter. Masses of bacilli in and around vessel walls. Giemsa $\times 780$.

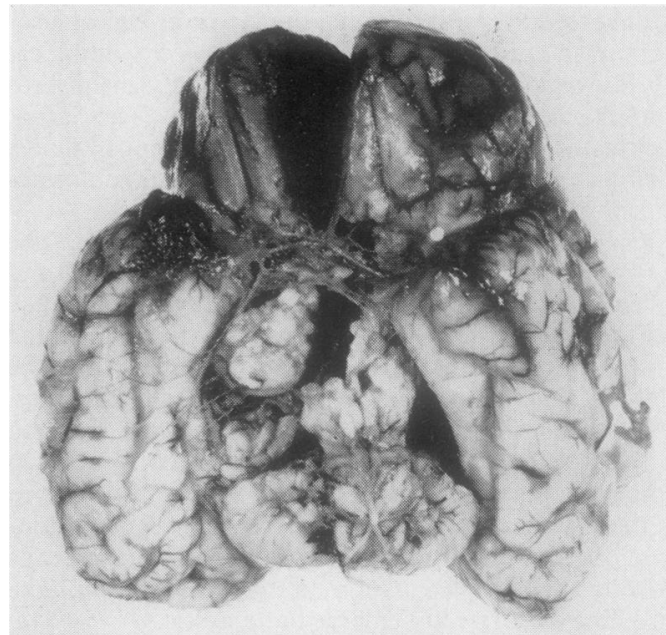

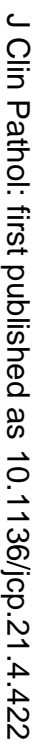

FIG. 4. Case 8. Brain after fixation. Inferior surface showing blackening of frontal lobes and disruption of cerebral peduncles.

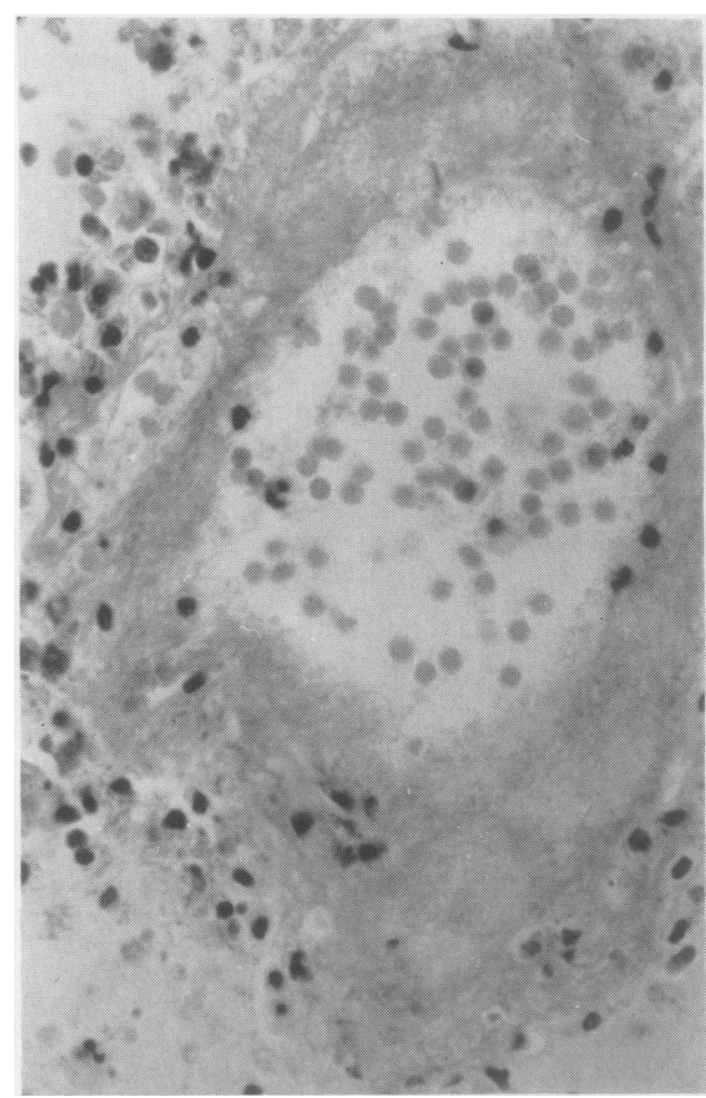

FIG. 6. Case 8. Cerebral white matter. Fibrinous necrosis $\stackrel{\mathbb{D}}{\stackrel{8}{2}}$ of vessel wall with perivascular exudate. Haematoxylin and eosin $\times 780$. 


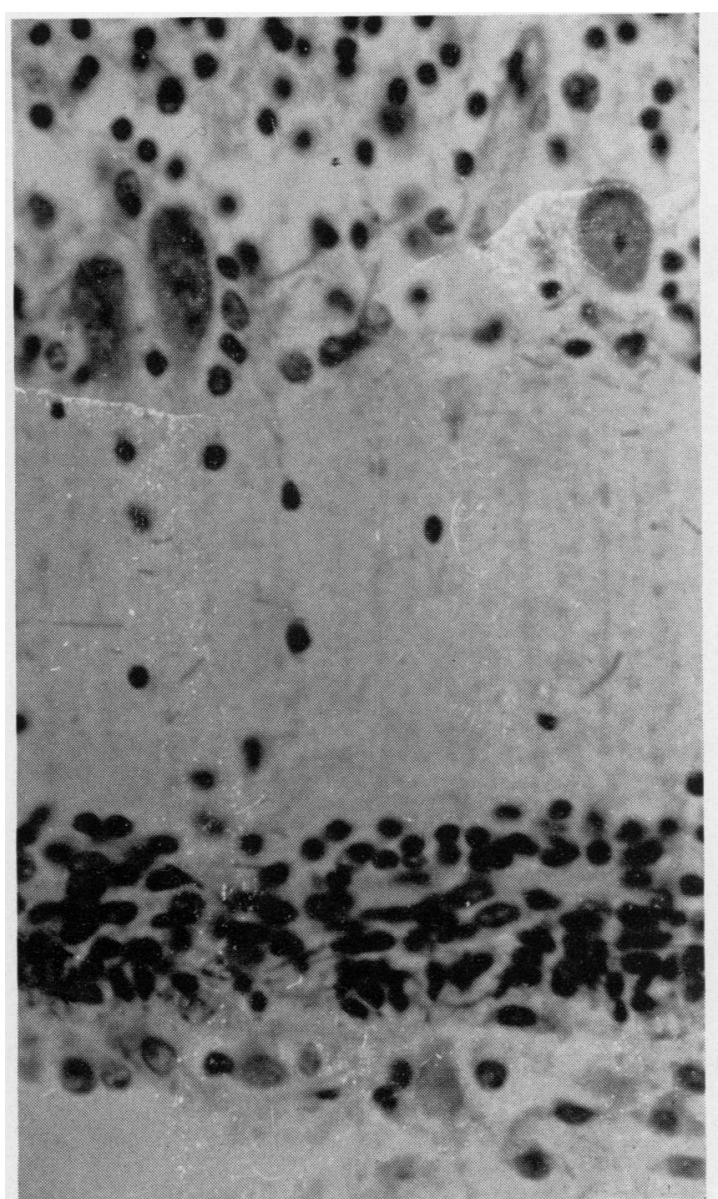

FIG. 7. Case 8. Bacilli in intact cerebellar cortex. Giemsa $\times 780$.

even in haematoxylin and eosin preparations. The hindbrain showed none of these features and cellular morphology was well retained; the only abnormality consisted of cytoplasmic swelling of many astrocytes in the white matter of the cerebellum and brainstem. Gram and Giemsa preparations showed enormous numbers of Gram-negative bacilli; they were especially abundant in the frontal lobes and around the vessels of the midbrain. Rather surprisingly there were also numerous filamentous bacilli throughout the otherwise normal cerebellum and brain stem. The lungs showed a few squames and macrophages together with small quantities of bile pigment in the air sacs.

The other twin Apart from slight icterus in the first few days the surviving twin remained well; birth weight was $1,857 \mathrm{~g}$. His weight gain was satisfactory and he attained the weight of $2,495 \mathrm{~g}$ upon discharge. Further progress of the surviving twin was not recorded as the mother did not take him to the follow-up clinic. The placenta was dichorionic and showed no unusual features. Histological examination of the placenta, membranes, and cords related to both twins showed no inflammatory changes or other features of note.

CASE 9 A female infant was born at 43 weeks to a primiparous mother. Pregnancy, labour, and delivery were normal. The infant weighed $3,359 \mathrm{~g}$. On the eighth day she became restless, irritable, and did not take her feeds. On the following day she kept moaning and would not sleep; she rolled her eyes from side to side and cried as though frightened; there were twitching movements of the head and she was very pale. On admission to the Birmingham Children's Hospital she was moribund, grey, cyanosed, apnoeic, and unresponsive but her peripheral circulation remained good. The anterior fontanelle was bulging and the umbilicus was septic. The pupils were fixed in mid-position and the discs were normal. The pulse was regular at $160 /$ minute. There was some respiratory stridor with indrawing of the ribs and moist sounds over both lungs. Lumbar puncture produced xanthochromic fluid which was under increased pressure and contained 3,850 polymorphonuclear leucocytes per cmm, protein $800 \mathrm{mg} \%$, and sugar $20 \mathrm{mg} \%$. Two Gram-negative bacilli were seen in smears and cultures provided a few colonies of Proteus mirabilis. Blood culture grew Proteus mirabilis and the pour plate blood culture showed 114 haemolytic colonies per millilitre of blood. Subcultures showed that these were all Proteus which had the same antibiogram as those cultured from the cerebrospinal fluid and which were sensitive to streptomycin, chloramphenicol, neomycin, ampicillin, and cephaloridine. The infant was treated with chloramphenicol, sulphadimidine, penicillin, amylobarbitone, and hydrocortisone but she failed to respond and died on the morning following admission.

Necropsy was performed 11 hours after death. Weight was $3,300 \mathrm{~g}$, length $52 \mathrm{~cm}$, head circumference $37 \mathrm{~cm}$. The anterior fontanelle was wide and slightly bulging. The umbilicus appeared to be clean and healthy. The umbilical vein was patent and unremarkable as was the ductus venosus. The left umbilical artery appeared to be normal. A segment of the right umbilical artery in its course alongside the bladder and within the pelvis was firm and swollen, with reddening of the surrounding tissues. Slicing of this segment showed pus within its lumen. Both lungs were firm and congested throughout. On reflecting the scalp the slightly bulging fontanelle was confirmed. Both the scalp and pericranium overlying the fontanelle had a dusky orange-black discoloration. There was a distinct, foul, fishy odour which was more intense when the skull was opened. Much of the cerebral hemispheres was blackish discoloured, extremely softened, and easily disrupted on attempted removal. Many of the cerebral veins contained firm thrombi but the cranial venous sinuses were clear. There was thick, dark-grey exudate in the interpeduncular cistern at the base of the brain and occluding the fourth ventricular foramina. The middle ears were clear. Slicing of the brain following fixation showed the same dark-grey colour throughout the cut surface except for a little cortical tissue in the right temporal lobe. The periventricular structures and the basal ganglia were grossly softened and easily 

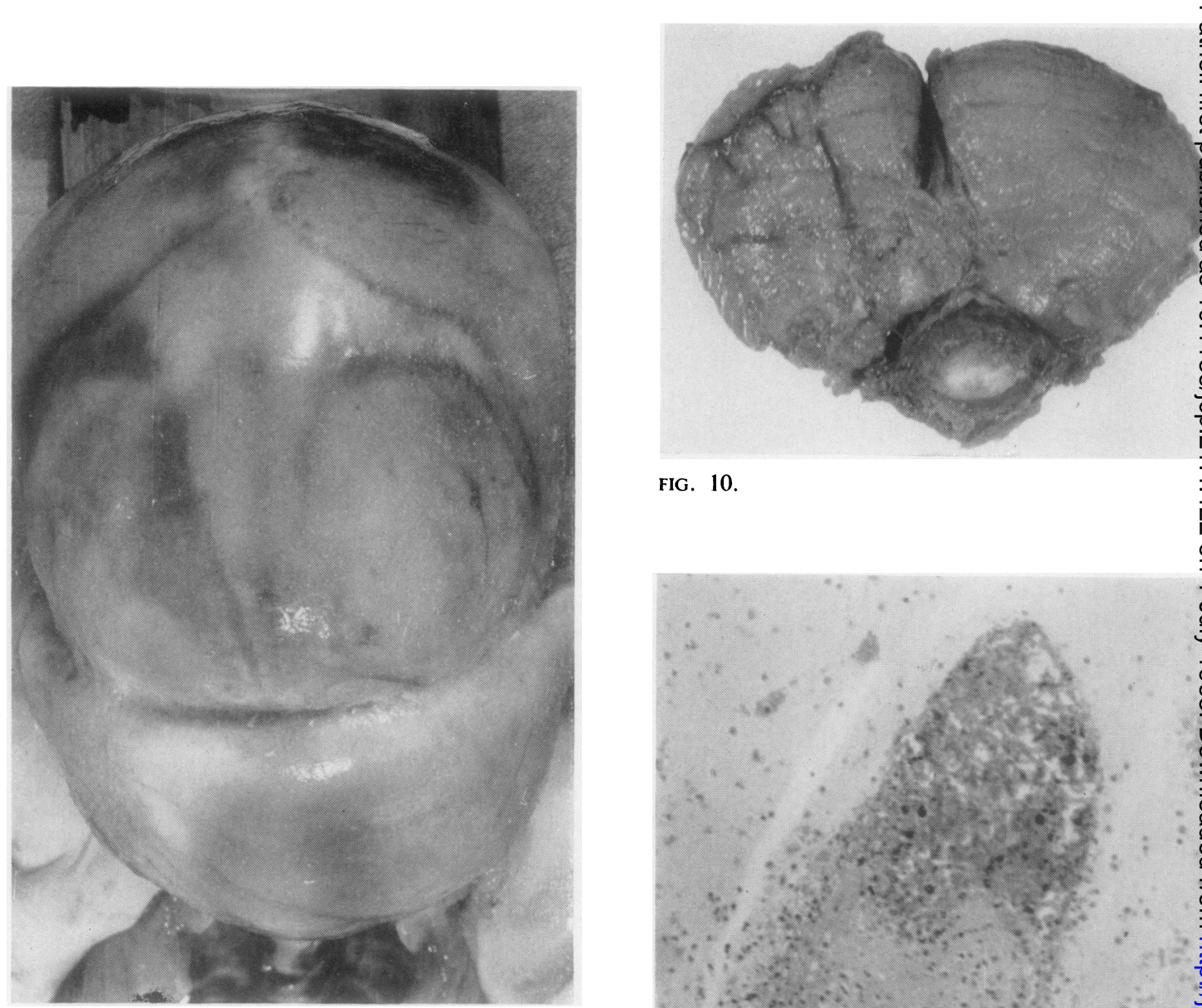

FIG. 10 .

FIG. 8 .

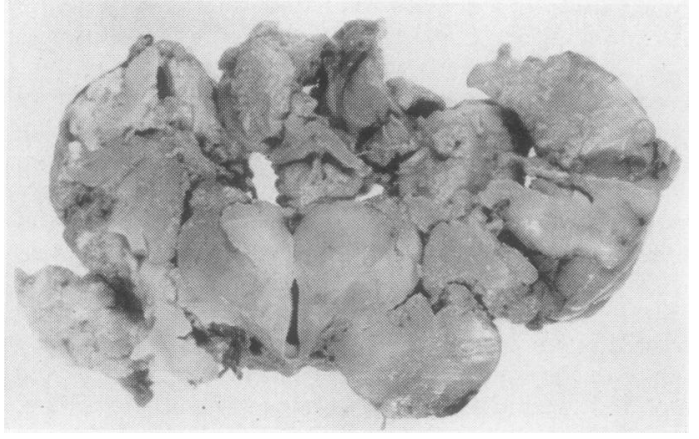

FIG. 9.

FIG. 10. Case 9. Exudate surrounding brain stem. venule with surrounding inflammatory exudate. Giemsw $\times 193$. 


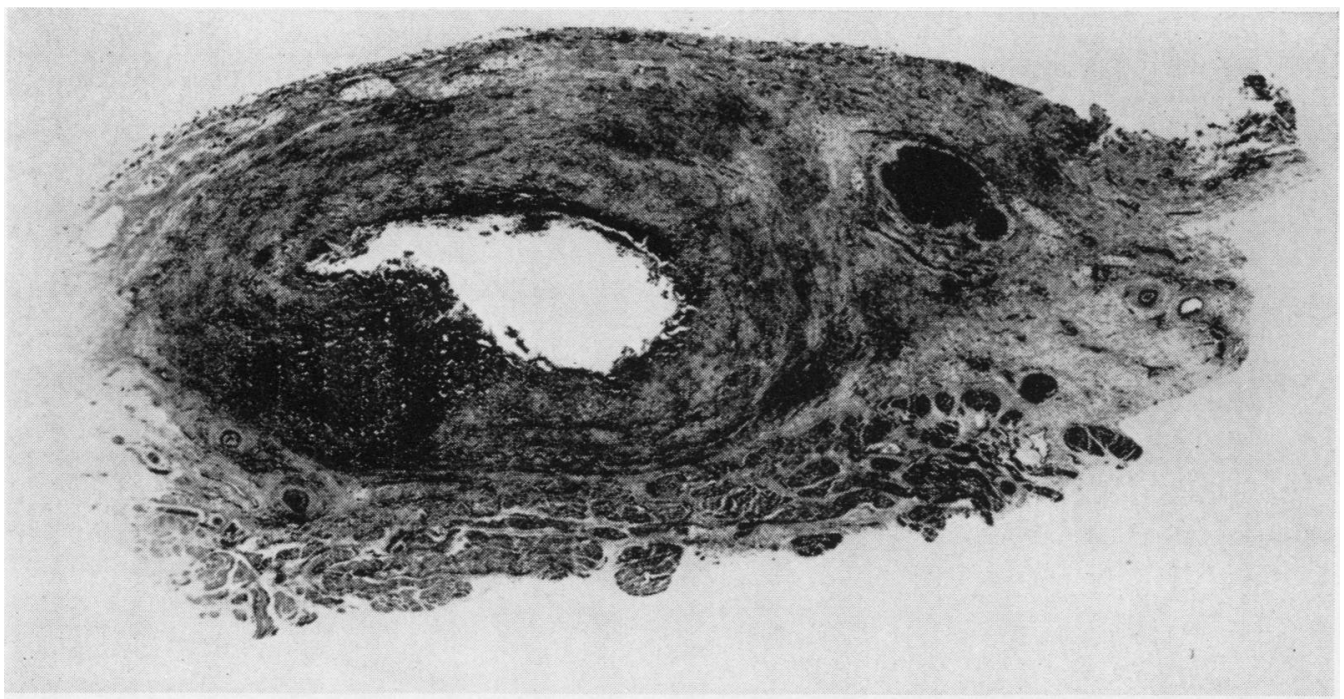

FIG. 12. Case 9. Right umbilical artery. The dark area represents an abscess in the wall. Haematoxylin and eosin $\times 6.5$.

disrupted. The hindbrain was similarly discoloured throughout but not softened.

Bacteriology A very heavy growth of Proteus was obtained from a meningeal swab, a heavy pure growth from the right umbilical artery and a heavy growth from an umbilical swab.

Histology Sections from three lobes of lung showed areas of pneumonia, apparently of recent origin with a sparse polymorphonuclear exudate. Organisms were not seen in Gram and Giemsa preparations. Two sections of the umbilicus showed complete epithelialization of the umbilical pit. One section included the umbilical vein which was almost totally occluded by aseptic granulation tissue. The other section from the centre of the umbilicus between the vein and arteries showed an acute abscess cavity lying immediately underneath the subepidermal granulation tissue. The umbilical abscess contained a few Gram-negative bacilli whilst the umbilical pit contained only a few Gram-positive cocci among desquamating cells. The right umbilical artery in its suprapubic segment showed acute inflammation of its wall extending into the surrounding fibro-adipose tissue and an acute abscess cavity which contained numerous Gram-negative rods. A block from the infraumbilical segment showed acute inflammatory infiltration involving the wall and the granulation tissue which partly occluded the lumen; the remains of the lumen were occupied by fibrino-purulent material. Similar changes were seen in the left umbilical artery at this level, but they were relatively slight. The inflammatory material within the two arteries near the umbilicus contained many Gram-negative rods. Sections of brain showed a polymorphonuclear and mononuclear exudate in the piaarachnoid over the cerebrum and hindbrain. Much of the cerebrum was frankly necrotic and there were many small cystic spaces of liquefaction. A zone of necrotic inflammatory cells surrounded many of the vessels, the walls of some of which were represented by structureless, eosinophilic rims of fibrinous appearance. There was no cellular infiltration of the intervascular necrotic parenchyma. All these areas showed many Gram-negative rods. The lateral ventricles showed acute exudate over the choroid plexus and considerable acute inflammatory infiltration in the subependymal zone.

The hindbrain showed none of the frank necrosis seen in the cerebral hemispheres, and the fourth ventricle showed no ependymitis. Many blood vessels, however, showed a narrow cuff of inflammatory cells in the Virchow-Robin spaces; this was especially so in the ventral half of the pons. There were numerous Gramnegative rods around these vessels and also in the intervening apparently normal parenchyma. Numerous organisms were also seen in the cerebellar folia even though the cortical cells appeared normal and there was no inflammatory change. The anterior lobe of the pituitary was normal and contained no organisms whilst the posterior lobe showed a peculiar homogeneous eosinophilic change of the matrix, pyknosis, and fragmentation of many nuclei and numerous Gram-negative rods.

CASE 10 A male infant was born at 32 weeks to a healthy 20-year-old primiparous mother. Pregnancy and labour were normal. Birth weight was $1,899 \mathrm{~g}$. For the first few days he did not suck well, had oedema of the eyes, hands, and feet, with slight cyanosis of the extremities and slight jaundice which cleared spontaneously. At 5 weeks he became irritable and cyanotic; he was unable to open his mouth and had rotary nystagmus, generalized stiffness, and an odd cry with wheezing respirations. He 
was thought to have meningitis and lumbar puncture produced bloody fluid which on culturing grew Klebsiella which were sensitive to tetracyclines, streptomycin, and chloramphenicol. Blood culture produced nonlactose fermenting bacilli which were members of the citrobacter group. He was treated with oxygen, chloromycetin, sulphadimidine, and cloxacillin but died about 20 hours after the onset of symptoms.

Necropsy was performed 35 hours after death. The body was that of a pale, premature infant weighing $2,340 \mathrm{~g}$ and measuring $49 \mathrm{~cm}$ in length, with a head circumference of $33 \mathrm{~cm}$. The umbilicus was well healed and clean. There was a foul smell both on reflecting the scalp and on opening the cranial cavity. Purulent exudate covered the cerebellum and both cerebral hemispheres which were softened and blackened. The lungs showed basal congestion. The abdominal contents, especially the umbilical vessels, were unremarkable.

Bacteriology Direct smears of the meninges showed pus cells and short, Gram-negative rods. Culturing showed these organisms to be non-lactose fermenting; they were not easy to classify but were subsequently labelled by the Salmonella Reference Laboratory as being members of the citrobacter group. These organisms were insensitive to penicillin, tetracyclines, erythromycin, sulphadimidine, and ampicillin.

In addition to a scanty growth of non-lactose fermenting bacilli a heavy growth of Lancefield group $\mathbf{A}$ haemolytic streptococci was obtained from a portion of lung.

Histology There were numerous Gram-negative, short rods scattered diffusely in the substance of the brain and in clumps within cerebral blood vessels. Haematoxylin and eosin preparations of the cerebral cortex showed vascular congestion and some exudate composed of pus cells and macrophages. The exudate was rather less marked than expected from naked eye appearances. The lungs showed extensive, intraalveolar, mononuclear exudate.

\section{DISCUSSION}

With one exception the cases described were either premature or dysmature neonates in whom there were symptoms and signs referrable to the respiratory and central nervous systems and in four of whom there was evidence of umbilical sepsis. Eight of the 10 infants succumbed within the first 10 days of life.

The characteristic features of Proteus meningoencephalitis were the smell and the gross and histological appearances of the cranial contents at necropsy. Primary infection by Proteus was established beyond doubt by bacteriological isolation of these organisms during life in six of the cases. Proteus organisms are motile and are known to flourish at room temperature, one of their characteristics being the ability to swarm under favourable conditions. For these reasons the possibility of secondary invasion by these organisms must be

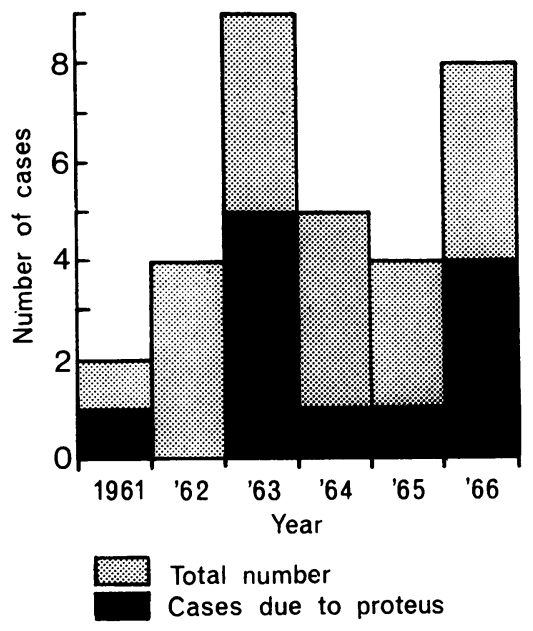

FIG. 13. Graph showing the number of cases of neonatal meningitis necropsied at the Children's Hospital, Birmingham, from 1961 to 1966. Two cases of Proteus meningitis that originated in the same nursery as case 9, but examined elsewhere, are also included. Case 10, which was not due to Proteus, is the case recorded in 1965.

considered in the three remaining cases in whicho Proteus was isolated from material obtained at necropsy.

As case 2 was necropsied less than 12 hours after death and heavy, though not pure growths of Proteus were obtained from the softened, discol oured, and offensive smelling brain and fronf heart blood, it was felt that the primary infection irg this case was due to Proteus. There was histologicap. evidence of meningitis and umbilical sepsis in case 5 and histological evidence of meningitis in case $8 \stackrel{3}{,}$ this evidence, together with bacteriological isolation of Proteus, seems strong evidence in favour of Proteus sepsis having been the primary conditio in both these cases too.

The remaining case has been included in order tฮึ illustrate that variants of $E$. coli may rarely bS responsible for similar necropsy appearances of the brain and that whilst there are characteristic pathog logical features by which Proteus meningeo encephalitis may be recognized, bacteriologicâ confirmation is necessary in every case.

The 10 cases described were encountered front 1961 to 1966 during which time, excluding case 8 with spina bifida, there were 30 necropsies of neonates with acute meningitis. Groover et al. (196\% found that Proteus was the cause in approximatel $4 \%$ of reported cases of neonatal meningitis. The

\section{.}


relatively high incidence of Proteus in necropsy cases of meningitis is an indication of the severity of this condition. There are few reports of successful treatment (Becker, 1962; Tolmas and Winter, 1957), and there have been no survivors in the Birmingham area.

During this period there have been only two other known cases in this area; they are included in the graph (Fig. 13) as they originated in the same nursery as case 9 . The graph shows two small clusters, one in 1963 and the other in 1966, indicating that epidemiological factors may be of importance in the aetiology. It is not intended to pursue this aspect here, but the finding of Proteus sepsis of the umbilicus or of the intraabdominal segments of the umbilical arteries in half of the cases is important. It indicates that this is commonly the portal of entry and possibly that the method used in the routine treatment of the umbilical stump may be a predisposing factor. This aspect is currently under further investigation.

I am indebted to Dr A. H. Cameron, the instigator of this paper, for all the encouragement and advice that he has given me in its preparation. I would like to thank Dr K. B. Rogers for his advice concerning the bacteriology on the 10 cases presented. The Salmonella Reference Laboratory, Central Public Health Laboratory, London, was responsible for finally labelling the causative organism in case 10.

I am grateful to Mr R. H. Bailey for technical assistance, Mr F. Mehta for photography, and Miss A. E. Arnold for secretarial assistance.

\section{REFERENCES}

Becker, A. H. (1962). Amer. J. Dis. Child., 104, 355

Groover, R. V., Sutherland, J. M., and Landing, B. H. (1961). New Engl. J. Med., 264, 1115.

Kaplan, M. Z., and Poweleit, A. C. (1949). Amer. J. Dis. Child., 77, 454.

Tolmas, H. C., and Winter, J. (1957). Ibid., 94, 574. 